\title{
Structural change of vortex patterns in anisotropic Bose-Einstein condensates
}

\author{
N. Lo Gullo, ${ }^{1}$ Th. Busch, ${ }^{1}$ and M. Paternostro ${ }^{2}$ \\ ${ }^{1}$ Department of Physics, University College Cork, Cork, Republic of Ireland \\ ${ }^{2}$ School of Mathematics and Physics, Queen's University, Belfast BTr 1NN, United Kingdom
}

(Dated: August 27, 2018)

\begin{abstract}
We study the changes in the spatial distribution of vortices in a rotating Bose-Einstein condensate due to an increasing eccentricity of the trapping potential. By breaking the rotational symmetry, the vortex system undergoes a rich variety of structural changes, including the formation of zigzag and linear configurations. These spatial re-arrangements are well signaled by the change in the behavior of the vortex-pattern eigenmodes against the eccentricity parameter. This behaviour allows to actively control the distribution of vorticity in many-body systems and opens the possibility to study interactions between quantum vortices over a large range of parameters.
\end{abstract}

PACS numbers: 67.85.De 03.75.Lm 47.32.cb

The superfluid nature of atomic Bose Einstein condensates (BECs) is one of the most striking manifestations of quantum mechanics on a macroscopic scale. Its telltale sign, the formation of quantized vortices, has been extensively studied in recent years and led to significant progress in understanding the phenomenon [1]. However, to create these topological defects one usually needs to break the rotational symmetry of the condensate. This requires a significant external disturbance through, for example, optical phase imprinting techniques [2] or stirring laser fields that allow to excite quadrupole-mode resonances [3]. The latter one is similar to the classical rotating bucket method that creates a vortex by rotating a bucket full of water and was first used in experimental studies of superfluid ${ }^{4} \mathrm{He}[4]$. The validity of such an analogy is certainly limited, given that the non superfluid component in superfluid ${ }^{4} \mathrm{He}$ experiences friction due to its relative motion with respect to the walls. As in the classical case, this implies a transfer of energy and angular momentum from the walls of the bucket to the superfluid part through the non-superfluid component. In a trapped BEC the latter is always negligible and the transfer has to be made through the excitation of normal modes, usually quadrupole ones. A BEC thus reacts to a large amount of angular momentum by creating many vortices with winding number equal to one [5], which arrange themselves in geometrically defined spatial patterns. At large vortex density, these structures mimic the celebrated Abrikosov lattice [6]. For harmonically trapped alkali condensates this was first observed in the seminal experiment by Abo-Shaeer et al. [3], where more than 100 vortices formed a triangular-shaped lattice with a few seconds lifetime.

Recently, numerical evidence has been provided that the vortex pattern of a $2 \mathrm{D} \mathrm{BEC} \mathrm{in} \mathrm{an} \mathrm{in-plane} \mathrm{anisotropic}$ rotating trap can undergo structural changes as a function of the eccentricity. Specifically, in Ref. [7] it has been shown that, for modest changes in the eccentricity, an off-line configuration (typical for an Abrikosov lattice) can change into a linear one. While this bears analogies with the case of ionic crystals [8], the characterization of structural changes in anisotropic and rotating BECs re- mains largely unexplored. Most of the existing literature focuses on the limit of large numbers of vortices for either a symmetric trap [9, 10] or very high angular frequencies, which leads to stripe-shaped vortex patterns [11 14]. Although the case of medium vorticity has been addressed, the role of external forces on the dynamics of the vortex structures still awaits a systematic approach [15 18]. Yet, understanding how vortices behave under external perturbations is a pre-requisite for harnessing the quantum properties of vortex patterns. Here we present a significant contribution to advance these aims by studying the behavior of finite-sized vortex patterns in 2D BECs confined within a rotating anisotropic trap. In particular we investigate in detail the effects of the eccentricity on the spatial distribution of the vortices. By minimizing the eccentricity-dependent interaction potential between vortices, we show that the vortex configuration

undergoes structural changes as the eccentricity parameter is varied. A hydrodynamical approach to the description of the superfluid motion allows us to identify the eigenmodes of the vortex-patterns and connect the appearance of discontinuities with the transition points between different structures. In fact, the modes suggest that the change in the equilibrium positions of the vortices is due to the re-arrangement of the superfluid velocity field.

\section{VORTEX PATTERN}

We consider the pattern of vortices in the ground state of a BEC held in a rotating trapping potential. The ground state is found by minimizing the energy functional [19]

$\mathcal{E}\left[\Psi, \Psi^{*}\right]=\int d^{3} \mathbf{r}\left[\frac{\hbar^{2}|\nabla \Psi|^{2}}{2 m}+V(\mathbf{r})|\Psi|^{2}+\frac{N g|\Psi|^{4}}{2}-\Psi^{*}(\boldsymbol{\Omega} \cdot \hat{\mathbf{L}}) \Psi\right]$

where $\Psi$ is the normalized order parameter of the condensate (its dependence on $\mathbf{r}$ is omitted for ease of notation), $V(\mathbf{r})$ is the trapping potential, $m$ is the atomic mass, $N$ is the number of atoms, $g=4 \pi \hbar^{2} a / m$ is the inter-atomic 
interaction energy volume determined by the s-wave scattering length $a, \boldsymbol{\Omega}$ is the rotation frequency vector of the condensate and $\hat{\mathbf{L}}$ is the angular momentum operator. The function $\Psi$ minimizing $\mathcal{E}$ has been studied both numerically and analytically under different working assumptions such as the Thomas-Fermi (TF) approximation [19], the lowest-Landau-level (LLL) approximation [20] or the limit of very weak interactions [16]. The first usually corresponds to the requirement of a very large number of particles, so that the kinetic energy associated with $\nabla\left|\Psi_{N S}\right|$ (with $\Psi_{N S}$ representing the nonsingular part of the order parameter) can be neglected in favor of the boson-boson interaction. In the LLL approximation, on the other hand, the main contribution to the energy stems from the centrifugal term and $\Psi$ is well described by means of single-particle wave-functions. Finally, in the limit of weak interactions the healing length becomes large and even under strong rotation only a small number of large vortices nucleate [16.

Here we consider a BEC in a harmonic trap rotating about its $z$-axis, which is also the direction of tightconfinement, so that $\Psi$ can be factorized into an axial part (the ground state of a harmonic potential) and an in-plane one, $\psi(x, y)$. We call $\omega_{j}(j=x, y)$ the trapping frequency along axis $j$ of the trap and introduce the eccentricity parameter $\lambda=\omega_{y} / \omega_{x}$.

We are now in a position to minimize $\mathcal{E}$ in the TF limit. For a set value of $0<\lambda \leq 1$, we call $\Omega_{N_{v}}(\lambda)$ the minimum angular frequency of the trap which allows for $N_{v}$ vortices in the state which minimizes $E\left[\Psi, \Psi^{*}\right]$, while $\mathbf{r}_{i}$ is the position of the $i^{\text {th }}$ vortex in the frame rotating with the condensate. By introducing $\left|\mathbf{r}_{i}\right|_{\lambda}^{2}=x_{i}^{2}+\lambda^{2} y_{i}^{2}$, the energy of the vortex pattern can be written as $U=U_{T}+U_{I}$ with [21]

$$
U_{T}=\frac{\pi \rho_{0}(\lambda)}{\left(1+\lambda^{2}\right)} \sum_{i=1}^{N_{v}}\left|\mathbf{r}_{i}\right|_{\lambda}^{2}, U_{I}=-\pi \rho_{0}(\lambda) \sum_{i=1}^{N_{v}} \sum_{j \neq i=1}^{N_{v}} \log \left|\mathbf{r}_{i}-\mathbf{r}_{j}\right| .
$$

Here, $\rho_{0}(\lambda)=\sqrt{2 \lambda / \pi}$ is the density of the condensate at the center of the trapping potential and minimizing these energies will determines the positions of vortices. In doing this, we will assume that the variations of $\lambda$ are accompanied by an adiabatic change of the angular frequency so that $\Omega_{N_{v}}(\lambda) \leq \Omega \ll \Omega_{N_{v}+1}(\lambda)$, which ensures that the wave-function minimizing the energy functional carries exactly $N_{v}$ vortices. The absence of a $\Omega$-dependent term from the expression of $U_{T}$ can be understood by considering that, in the TF limit, the centrifugal force is proportional to the restoring term for $\Omega \approx \Omega_{N_{v}}(\lambda)$ (see Eq. (3.4) in Ref. [14]), so that they sum up to a quantity which is independent of $\Omega$. In order to quantitatively asses the deviations of the vortex pattern from the Abrikosov-like lattice [7], we first show how the distances of the vortices from the tight trapping direction vary against the eccentricity $\lambda$. Two representative cases $\left(N_{v}=7,8\right)$ of the general dynamics are shown in Fig. 1] the pattern of vortices corresponding to values of $\lambda$ larger than a critical threshold $\lambda_{L}$ (in general a function of $N_{v}$ ) (a)

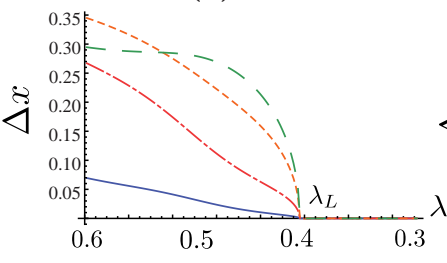

(b)

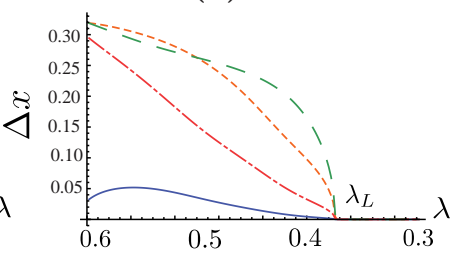

FIG. 1. (Color online) Distance $\Delta x$ of the vortices from the soft trapping axis (in units of $\sqrt{2 N g \Omega / \hbar \omega_{x}}$ ) against the eccentricity of the trap, $\lambda$. We show the cases of $N_{v}=7$ and 8 (panel (a) and (b) respectively) and plot only the changes in positions of four vortices in the lattice (the association with the curves is irrelevant), with the remaining showing analogous behavior. At $\lambda=\lambda_{L}$ the vortices suddenly align along the $\mathrm{y}$-axis $(\Delta x=0)$. We have used a BEC of $10^{6}{ }^{87} \mathrm{Rb}$ atoms with a scattering length $a=5.23 \times 10^{-9} \mathrm{~m}$ in a trap of frequencies $\omega_{z} / 2 \pi=100 \mathrm{~Hz}$ and $\sqrt{\omega_{x} \omega_{y}} / 2 \pi=50 \mathrm{~Hz}$ (independent of $\lambda)$.
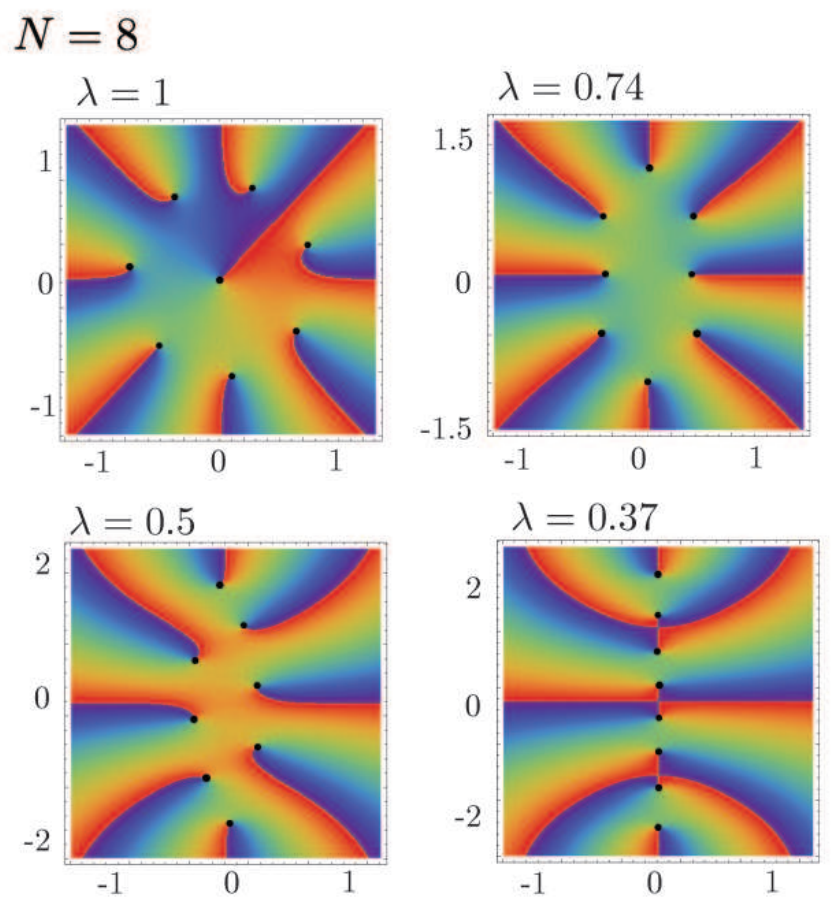

FIG. 2. (Color online) Phase distribution of the condensate carrying a vortex-lattice in the $x-y$ plane with $N_{v}=8$ for different values of $\lambda$. The black dots mark the positions of the vortices (in units of $\sqrt{2 N g \Omega / \hbar \omega_{x}}$ ) The appearance of different structural vortex patterns is clearly visible.

abruptly collapses to an all-aligned configuration.

However, looking at the distance of the vortices from the soft axis only gives limited information about the actual vortex pattern and we show in Figs. 2 and 3 the full position distribution for different numbers of vortices and different values of $1 \geq \lambda>\lambda_{L}$. Two more structurally distinct configurations become evident from this and let 
$N=7$
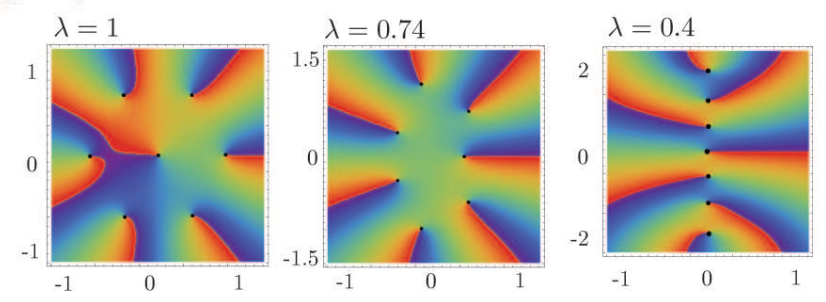

FIG. 3. (Color online) Phase distribution of the condensate carrying a vortex-lattice in the $x-y$ plane with $N_{v}=7$ for different values of $\lambda$. All other values are as in Fig. 2

us first consider the case of an even number of vortices (shown in Fig. 2): starting from an Abrikosov-like pattern at zero eccentricity $(\lambda=1)$, the first structural change at $\lambda=\lambda_{C}$ witnesses the central vortex being displaced so as to join the ring formed by the outer ones. A further reduction of $\lambda$ leads to a second threshold value, $\lambda_{Z}$, at which the mirror symmetry is broken and a zigzag pattern is formed. The situation is different for an odd number of vortices, where a parity effect leads to the Abrikosov-to-ring and ring-to-zig-zag transitions becoming degenerate: from full isotropy the lattice re-arranges directly into a zig-zag pattern at $\lambda=\lambda_{Z}$, see Fig. 3. For even, as well as odd number of vortices a further reduction in $\lambda$ makes the vortices align along the weak trapping direction, as already observed in Fig. 10 The situation is even richer for a larger (but finite) number of vortices. Let us consider, for instance, a system consisting of 18 vortices (see Fig. (4). As shown in panel (a), at $\lambda=1$ they arrange in a pattern with a single vortex at the centre of the trap and two concentric rings surrounding it. By decreasing $\lambda$ we first observe an Abrikosov-to-ring structural change involving the inner ring (made out of six vortices) and the central vortex, similar to the one described above (see panel (b)). By further decreasing $\lambda$, the vortices in the newly formed inner ring start joining the outer one (see panel (c)) before forming a zig-zag pattern (panel (d)). Finally, the transition into a linear structure occurs (not shown).

Let us briefly compare the vortex patterns we have just discussed with the ones presented in Refs. [11, 13]. In these works the authors address the case of an asymmetric trapping potential in the fast rotation limit, $\Omega \rightarrow$ $\min \left(\omega_{x}, \omega_{y}\right)$. In this case the condensate background cloud is stretched along the direction of weak confinement and assumes a stripe-like shape. Because of the symmetry of such a system vortices have to enter the cloud in rows and the possible geometries are given by whether or not vortices between different rows are aligned. In the case of fast rotating traps the solution is determined by single particle states and if the vorticity exceeds the number of atoms in the system, the existing lattice melts and a highly correlated state emerges. In contrast, we are dealing with a fixed number of vortices in the limit
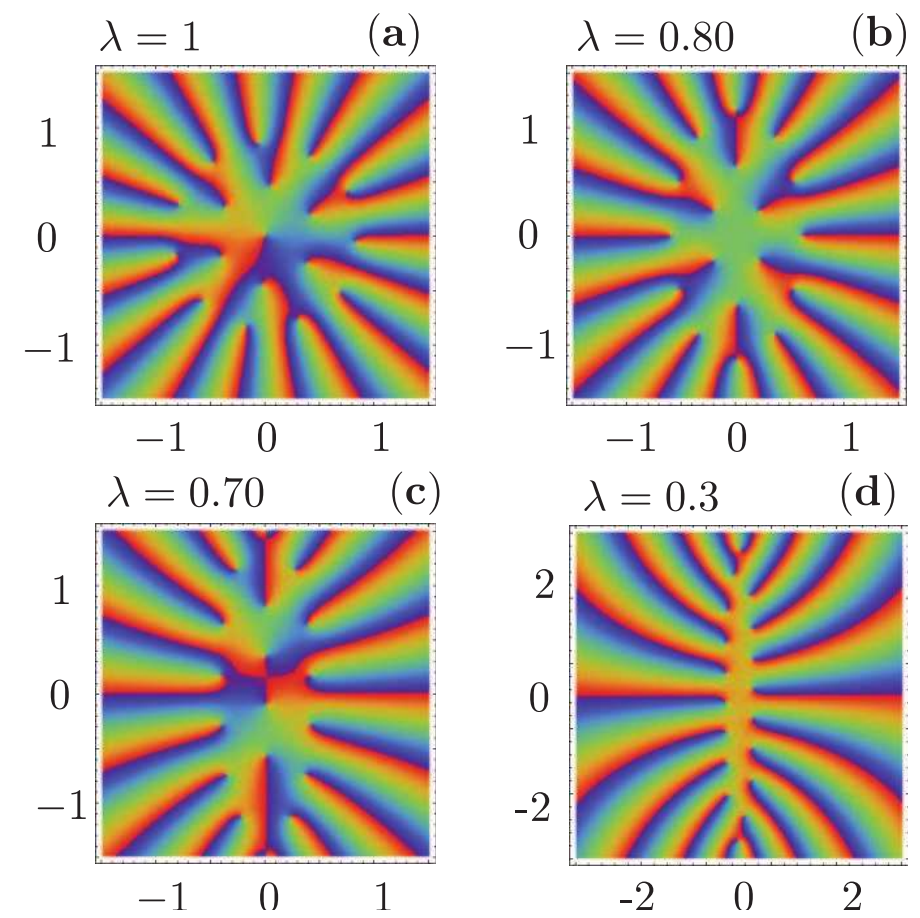

FIG. 4. (Color online) Phase distribution of the condensate carrying a vortex-lattice in the $x-y$ plane with $N_{v}=18$ for different values of $\lambda$. The black dots mark the positions of the vortices (in units of $\sqrt{2 N g \Omega / \hbar \omega_{x}}$ ).

where the interaction energy dominates the centrifugal one. For such systems the healing length is much smaller than any other characteristic length of the system, which, as we have shown, leads to a number of possible patterns with well localised singularities. Transitions between these patterns are then determined by the interplay between the trapping potential and the interaction energy between the vortices.

\section{SUPERFLUID HYDRODYNAMICS}

In this Section we will explore the structural transitions in detail by looking at the change in the superfluid motion of the condensate. This is analogous to an argument used by Fetter in Ref. [15], where superfluid motion in an elliptical and rectangular cylinder was studied. While both the energy and angular momentum of the system were found [15], the existence of a threshold value for the angular velocity above which the configuration with one vortex is energetically favorable was shown. It is important to stress that in our case the vortex-lattice configuration found by minimizing Eq. (2) does not represent, in general, a rigid pattern, due to the perturbations introduced into the system by the eccentricity. This can 
(a)

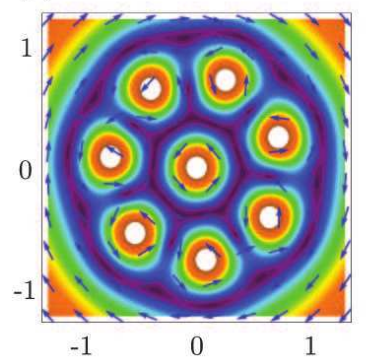

(c)

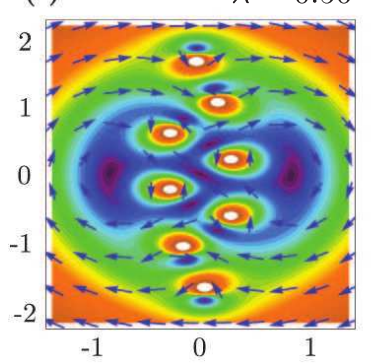

(b)

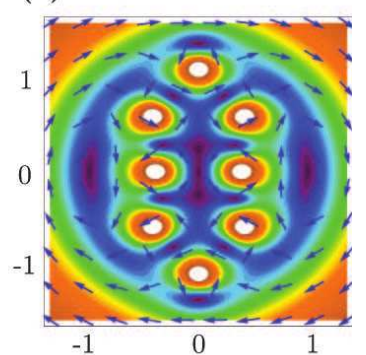

(d)

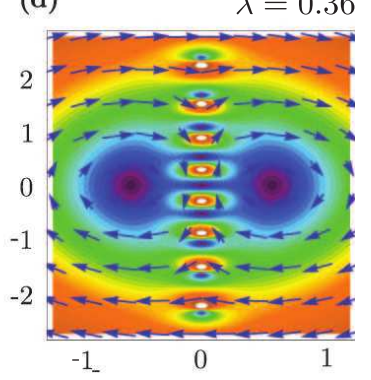

FIG. 5. (Color online) Superfluid velocity field in the rotating frame for $N_{v}=8$ (other parameters as in Fig. 1). From (a) to (d) the asymmetry parameter is given by $\lambda=1,0.76,0.56,0.36$. Dark purple regions correspond to zero velocity and the velocities close to the vortex cores are not shown on the chosen color-map.

be seen by recasting the trapping potential as

$V_{\lambda}(\mathbf{x}) \equiv V_{s}(\mathbf{x})+V_{Q}(\lambda, y)=\frac{1}{2} m \omega_{x}^{2}\left(x^{2}+y^{2}\right)+\frac{1}{2} m \omega_{x}^{2}\left(\lambda^{2}-1\right) y^{2}$

and recognizing $V_{Q}(\lambda, y)$ as a term exciting quadrupole modes. Thus the background condensate and the vortex pattern are not stationary.

The free energy of the rotating BEC is now given by $F_{N_{v}}=E_{N_{v}}(\Omega, \lambda)+U_{T}+U_{I}$ where $U_{T, I}$ are defined by Eq. (2) and $E_{N_{v}}(\Omega, \lambda)$ is an energy term that does not depend on the vortex configuration and whose detailed form is not essential for our discussions. By calling $\left\{\mathbf{r}_{i}^{0}\right\}\left(i=1, . ., N_{v}\right)$ the vortex positions which minimize Eq. (2) for a set number of vortices, we have the condition $\left.\nabla_{j} F_{N_{v}}\right|_{\left\{\mathbf{r}_{i}^{0}\right\}}=0$, where $\nabla_{j} \equiv\left(\partial_{x_{j}}, \partial_{y_{j}}\right)$ and where we have used the subscript $j$ to represent the coordinates of the $j^{\text {th }}$ vortex. In the rotating frame, a vortex has a velocity $\boldsymbol{v}_{j}$ such that

$$
\left.\nabla_{j} F_{N_{v}}\right|_{\mathbf{r}_{j}} \cdot \boldsymbol{v}_{\mathbf{r}_{j}}=0
$$

which implies the absence of dissipation, as expected from particles moving in a superfluid. A solution to this equation is given by $\boldsymbol{v}_{\mathbf{r}_{j}}=\alpha\left(\left.\nabla_{j}^{\frac{1}{j}} F_{N_{v}}\right|_{\mathbf{r}_{j}}\right)$ with $\nabla_{j}^{\perp} \equiv\left(\partial_{y_{j}},-\partial_{x_{j}}\right)$, where $\alpha$ is the amplitude of the velocity field. Its value

$$
\alpha=a_{h o} \frac{\sqrt{\Omega \omega_{x}}}{\pi \rho_{0}(\lambda)} \quad\left(\text { with } a_{h o}=\sqrt{\frac{\hbar}{m \omega_{x}}}\right)
$$

is found by comparing it with the velocity field $(\hbar / m) \nabla S-\boldsymbol{\Omega} \times \mathbf{r}_{j}$ in the rotating frame. In this expression, $S=S_{0}+\sum_{i \neq j}^{N_{v}} \theta_{i}$ is the phase of the order parameter as seen by the $j^{\text {th }}$ vortex, $\tan \theta_{j}=\left(y-y_{j}\right) /\left(x-x_{j}\right)$ specifies the polar angle of a reference frame centered on the $j^{\text {th }}$ vortex core [19] and

$$
S_{0}=-\frac{m \Omega\left(1-\lambda^{2}\right)}{\hbar\left(1+\lambda^{2}\right)} x y
$$

is the vortex-free phase of the BEC at position $(x, y)$.

In Fig. 5 we show the magnitude of the velocity field for $N_{v}=8$ in a frame which rotates rigidly with the trap. The value of $\lambda$ decreases from panel (a) to (d) and the arrows show the flow directions with the magnitude being encoded in the color. In the dark (dark purple) regions the velocity field vanishes, i.e. the superfluid moves at the trap angular velocity. For no eccentricity [panel (a)] the vortex pattern rotates rigidly with the trap potential since the velocity field at the vortex positions (when the vortex itself is not present) vanishes in the rotating frame. It is worth noticing that outside the vortex pattern particles flow with a different velocity. This is at the origin of the imperfect rigid-body rotation of finite-sized vortex patterns in isotropic traps. By increasing the eccentricity [panel (b)-(d)] the rigid body behavior is lost and the vortex pattern is no longer a steady solution [7], since the continuous rotation of the trap increases the angular momentum of the system. However, the condition $\Omega \in\left[\Omega_{N_{v}}, \Omega_{N_{v}+1}\right.$ [ on the angular velocity fixes the number of vortices in the condensate $N_{v}$. The only possibility for the system to react is to move the vortex cores to accommodate the angular momentum. In a real system, heating and dissipation would eventually lead to the crystallization of the vortex pattern or the transition to a turbulent regime [22].

\section{VORTEX LATTICE MODES}

A quantitative confirmation of the abrupt nature of the structural changes can be found by studying the eigenmodes of the vortex pattern [18]. We take a set of small displacements $\left\{\delta \mathbf{r}_{i}\right\}$ from the equilibrium configuration $\left\{\mathbf{r}_{i}^{0}\right\}$ and write

$$
\delta \boldsymbol{v}=\left(\delta v_{\mathbf{r}_{1}}^{x}, \delta v_{\mathbf{r}_{1}}^{y}, \ldots, \delta v_{\mathbf{r}_{N_{v}}}^{x}, \delta v_{\mathbf{r}_{N_{v}}}^{y}\right),
$$

so that the vortex cores velocities in the rotating frame become $\delta \boldsymbol{v} \simeq \mathbf{A} \cdot \delta \mathbf{r}$. Here A is a $2 N_{v} \times 2 N_{v}$ matrix whose $j^{\text {th }}$ row is found by expanding the velocity field $\boldsymbol{v}_{\mathbf{r}_{j}}=\left.\alpha \nabla_{j}^{\perp} F_{N_{v}}\right|_{\mathbf{r}_{j}}$ around each $\mathbf{r}_{i}^{0}$. This gives

$$
\mathbf{A}_{j}=\alpha \sum_{i}\left[\partial_{x_{i}}\left(\nabla_{j}^{\perp} F_{N_{v}}\right) \hat{x}_{i}+\partial_{y_{i}}\left(\nabla_{j}^{\perp} F_{N_{v}}\right) \hat{y}_{i}\right]_{\left\{\mathbf{r}_{i}^{\mathbf{0}}\right\}},
$$

where $\alpha$ is determined as before. We now numerically diagonalize $\mathbf{A}$ for a set number of vortices. The eigenvalues $\alpha_{l}\left(1 \leq l \leq 2 N_{v}\right)$ of $\mathbf{A}$ represent the rate at which 


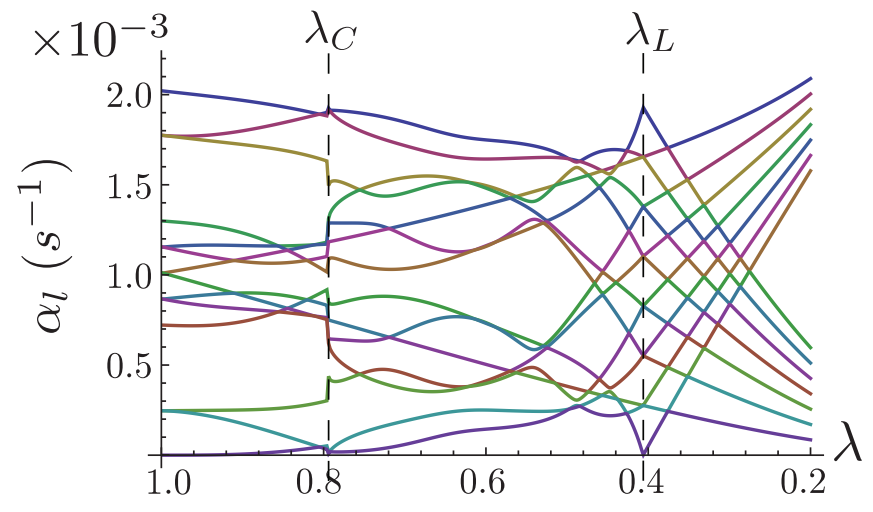

FIG. 6. (Color online). Spectrum of a BEC with $N_{v}=7$ vortices against the eccentricity $\lambda$. The points $\lambda_{C, L}$ where the vortex pattern undergoes a structural change are visible.

vortices start moving from $\left\{\mathbf{r}_{i}^{0}\right\}$ once they are displaced by the corresponding eigenvector $\delta \mathbf{r}^{l}$. We note that the eigenmodes are related by

$$
\alpha_{n}(\lambda)+\alpha_{2 N_{v}-n}(\lambda)=C(\lambda) \quad\left(0<n \leq N_{v}\right)
$$

and the corresponding eigenvectors are mutually orthogonal. The constant $C(\lambda)$ depends on the system parameters but, remarkably, is independent of the pair of eigenvectors considered. A typical spectrum for $N_{v}=7$ is shown in Fig. 6. At two specific values of $\lambda$ the eigenmodes show non-continuous behavior, beside the appearance of a null eigenvalue. These points can be connected to the structural transition points: $\lambda_{C}$ signaling the Abrikosov-to-ring transition and $\lambda_{L}$ the zig-zag-tolinear one. At any other value of $\lambda$ the eigenmodes are positive confirming our previous point on the non-steady nature of the vortex patterns in the rotating frame. However, the exact value of $\lambda$ at which the lowest eigenvalue first deviates from zero is found to grow with the number of vortices. The corresponding eigenvector corresponds to displacements of the vortex positions along the tangent to the vortex ring, i.e. a rotation of the vortex pattern produces no effect. In fact, it is not possible to clearly discriminate the eigenmodes of a finite-size lattice with a small number of vortices from the phonon modes of the background condensate: rotating an anisotropic trap excites Bogoliubov modes in the BEC, which have a strong influence on the vortex pattern [23]. The link between Bogoliubov modes and changes in the properties of the vortex matter has already been explored in relation to vortex-pattern formation and instability [24]. Moreover, in Ref. [25] stability of vortex clusters (comprising both vortices and anti-vortices) in a non rotating anisotropic trap has been studied by looking at the Bogoliubov modes. The dynamics induced between the background cloud and the vortex matter has there been shown to be not separable.

\section{CONCLUSIONS}

We have studied the structural transitions induced in a finite vortex-lattice by an increasing degree of eccentricity of a rotating BEC. An Abrikosov-like arrangement undergoes a sequence of symmetry-breaking processes that push it towards a linear arrangement of vortices. Such modifications, witnessed and understood in terms of background superfluid motion, are well signaled by the eigenmodes of the vortex-lattice. By addressing the case of a finite lattice, our work complements and extends the existing literature on vortex instabilities and arrangements in rotating BECs and provides interesting insight into the many-body properties of a mesoscopic quantum system. Our analysis is not limited to BECs: vortex-like excitations exist in superconducting films, Josephson-junction arrays and dislocation pairs in the theory of $2 \mathrm{D}$ melting [26]. Inter-vortex potentials depending logarithmically on the distance between two vortices, similar to Eq. (2), have been observed in thin superconducting films [27]. Vortex lattices in thin films under magnetic fields have been shown to take the form of discrete rows [28]. Strong analogies between the dynamics of vortex lattices and Josephson-junction arrays hold due to the charge-vortex duality [29], thus giving our results a generality and interest that goes beyond the cases addressed here.

\section{ACKNOWLEDGMENTS}

We thank G. Morigi and W. Bao for helpful discussions and invaluable help. NLG thanks G. Pucci for the Mini-amo project. This work was supported by SFI under grant numbers 05/IN/I852 and 05/IN/I852 NS, IRCSET through the Embark Initiative (RS/2000/137) and EPSRC (E/G004579/1).
[1] A.L. Fetter, Rev. Mod. Phys. 81, 647 (2009).

[2] M.R. Matthews, B. P. Anderson, P. C. Haljan, D. S. Hall, C. E. Wieman, and E. A. Cornell, Phys. Rev. Lett. 83, 2498 (1999); A.E. Leanhardt, ,A. Grlitz, A. P. Chikkatur, D. Kielpinski, Y. Shin, D. E. Pritchard, and W. Ketterle 89, 190403 (2002).

[3] K.W. Madison, F. Chevy, W. Wohlleben, and J. Dalibard, Phys. Rev. Lett. 84, 806 (2000); J.R. Abo-Shaeer,
C. Raman, J. M. Vogels and W. Ketterle, Science 292, 476 (2001).

[4] E.L. Andronikashvili, J. Phys. USSR 10, 201 (1946); A.J. Leggett in Low Temperature Physics, M. Hoch and R. Lemmer eds. (Springer, Berlin, 1992).

[5] J. Kim and A.L. Fetter, Phys. Rev. A70, 043624 (2004).

[6] A.A. Abrikosov, J. Exp. Theoret. Phys. 32, 1147 (1957). 
[7] S. McEndoo and Th. Busch, Phys. Rev. A79, 053616 (2009); ibid. 82, 013628 (2010). There the eccentricity parameter has been chosen as $1 / \lambda$.

[8] G. Birkl, S. Kassner, H. Walther, Nature 357, 310 (1992); I. Waki, S. Kassner, G. Birkl, and H. Walther, Phys. Rev. Lett. 68, 2007 (1992); J.P. Schiffer, ibid. 70, 818 (1993); G. Morigi and S. Fishman, ibid. 93, 170602 (2004).

[9] I. Coddington, P. Engels, V. Schweikhard, and E. A. Cornell, Phys. Rev. Lett.91, 100402 (2003).

[10] G. Baym, Phys. Rev. Lett.91, 110402 (2003); T. Mizushima, Y. Kawaguchi, K. Machida, T. Ohmi, T. Isoshima, and M. M. Salomaa, ibid. 92, 060407 (2004).

[11] S.I. Matveenko, D. Kovrizhin, S. Ouvry, and G.V. Shlyapnikov, Phys. Rev. A80, 063621 (2009).

[12] A. Aftalion, X. Blanc, N. Lerner, Phys. Rev. A79, 011603 (2009).

[13] P. Sánchez-Lotero, J.J. Palacios, Phys. Rev. A72, 043613 (2005).

[14] A.L. Fetter Phys. Rev. A75 , 79013620 (2007).

[15] A.L. Fetter, J. Low Temp. Phys. 16, 533 (1974).

[16] M.Ö. Oktel, Phys. Rev. A69, 023618 (2004).
[17] J.W. Reijnders and R.A. Duine, Phys. Rev. A71, 063607 (2005).

[18] L.J. Campbell, Phys. Rev. A24, 514 (1981).

[19] Y. Castin and R. Dum, Eur. Phys. J. D 7, 399 (1999); A. Aftalion and Q. Du, Phys. Rev. A64, 063603 (2001).

[20] L.D. Landau and E.M. Lifshitz, Statistical Physics (Pergamon, Oxford, 1958).

[21] R. Ignat and V. Millot, J. Funct. Anal. 233, 260 (2006); Rev. Math. Phys. 18, 119 (2006).

[22] N.G. Parker and C.S. Adams, Phys. Rev. Lett.95, 145301 (2006) .

[23] E. Kozik and B. Svistunov, Phys. Rev. B72, 172505 (2005).

[24] C. Lobo, A. Sinatra and Y. Castin, Phys. Rev. Lett.92, 020403 (2004).

[25] J. Stockhofe, S. Middelkamp, P.G. Kevrekidis, P. Schmelcher Eur. Phys. Lett. 93, 20008 (2011).

[26] B.I. Halperin and D.R. Nelson, J. Low. Temp. Phys. 36, 599 (1979); K. Mullen, Phys. Rev. B 60, 4334 (1999).

[27] J. Pearl, in Low temperature physics LT9, J.G. Daunt, D.O. Edwards, F.J. Milford and M. Yagub eds. (Plenum, New York, 1965).

[28] D.A. Luzhbin, Phys. Solid State 43, 1823 (2006).

[29] C. Bruder, R. Fazio, G. Schön, Ann. der Phys. 14, 566 (2005). 